\title{
The Exponentiated Burr XII Distribution: Moments and Estimation Based on Lower Record Values
}

\author{
Devendra Kumar ${ }^{1 *}$, Jagdish Saran ${ }^{2}$ and Neetu Jain ${ }^{2}$ \\ ${ }^{1}$ Department of Statistics, Central University of Haryana, India \\ ${ }^{2}$ Department of Statistics, University of Delhi-110007, India \\ *Corresponding Author: email address: devendrastats@gmail.com
}

Received: 15th July 2015/ Revised: 15th March 2017 / Accepted: 25th August 2017

CIAppStat-SL2017

\section{ABSTRACT}

In this paper, we have established several explicit expressions and recurrence relations for single and product moments of $k$-th lower record values from exponentiated Burr XII distribution. Two characterizing results of exponentiated Burr XII distribution has been obtained by using the recurrence relation for single moments and conditional expectation based on lower record values. The method of maximum likelihood is adopted for estimating the model parameters based on $k$-th lower record values. We carried out Monte Carlo simulations to compare the performances of the proposed methods and providing one real data case study for illustration of the results obtained.

Keywords: Lower record values, single and moments, recurrence relations, exponentiated Burr XII distribution, characterizations, maximum likelihood estimates, Simulation

\section{Introduction}

Record values find extensive applications in many real life situations involving data relating to weather, sport, economics, life testing studies and so on. There are several situations like Guinness World Records where only record values are observed. News items like fastest time taken to recite the periodic table of the elements, shortest ever tennis matches both in terms of number of games and duration in terms of time, fastest indoor marathon, longest time to hop on one foot, etc are of immense interest to people. Several attempts are made to make a record and records are made only when attempts are successful. Usually, we do not get the data on all of the attempts made to break the records around the world. The data that we have are the records. Another example is the situation in the

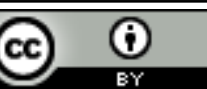


assessment of glucose level among diabetic patients, the researchers may be interested to study the behaviour of the ordered records of glocodine. Also, there are several situations where the lower record values are of special interest. For example, if various voltages of equipment are considered, only the voltages less than the previous one can be recorded. These recorded voltages are the lower record value sequence. Many scientists specially the statisticians have become interested in record values over the past 60 years or so since 1952 when Chandler (1952) first studied the distributions of lower records, record times and interrecord times for iid sequences of random variables. There are hundreds of papers and several books published on record-breaking data and its distributional properties see, for instance, Chandler (1952), Feller (1966), Resnick (1973), Shorrock (1973), Glick (1978), Nevzorov (1987), Balakrishnan and Ahsanullah (1995), Ahsanullah (1995), Kumar (2012, 2015, 2016), Kumar and Kulshrestha (2013) and Kumar and Saran (2014), Kumar et al. (2015) and so on.

Let $\left\{X_{n}, n \geq 1\right\}$ be a sequence of independent and identically distributed (i.i.d.) random variables with cumulative distribution function $c d f F(x)$ and probability density function $(p d f) f(x)$. The $j$-th order statistic of a sample $\left(X_{1}, X_{2}, \ldots, X_{n}\right)$ is denoted by $X_{j: n}$. For a fixed $k \geq 1$, we define the sequences $\left\{L^{(k)}(n), n \geq 1\right\}$ of $k-$ th lower record times of $X_{1}, X_{2}, \ldots$ as follows:

$$
\begin{aligned}
& L^{(k)}(1)=1 \\
& L^{(k)}(n+1)=\min \left\{j>L^{(k)}(n): X_{k: L^{(k)}(n)+k-1}>X_{k: j+k-1}\right\} .
\end{aligned}
$$

The sequence $\left\{Y_{n}^{(k)}, n \geq 1\right\}$ with $Y_{n}^{(k)}=X_{L^{(k)}(n)}, n=1,2, \ldots$ is called the sequence of $k-$ th lower record values of $\left\{X_{n}, n \geq 1\right\}$.

For $k=1$ and $n=1,2, \ldots$, we write $L^{(1)}(n)=L_{n}$. Then $\left\{L_{n}, n \geq 1\right\}$ is the sequence of lower record times of $\left\{X_{n}, n \geq 1\right\}$. For convenience, we shall also take $Y_{0}^{(k)}=0$. Note that for $k=1$ we have $Y_{n}^{(1)}=X_{L_{n}}, n \geq 1$ which are lower record values of $\left\{X_{n}, n \geq 1\right\}$. Moreover $Y_{1}^{(k)}=\min \left\{X_{1}, X_{2}, \ldots, X_{k}\right\}=X_{1: k}$ (Ahsanullah, 1995).

The joint $p d f$ of $k-$ th lower record values $Y_{1}^{(k)}, \ldots, Y_{n}^{(k)}$ can be given as 


$$
\begin{array}{r}
f_{Y_{1}^{(k)}, \ldots, Y_{n}^{(k)}}\left(x_{1}, x_{2}, \ldots, x_{n}\right)=k^{n}\left(\prod_{i=1}^{n-1} \frac{f\left(x_{i}\right)}{F\left(x_{i}\right)}\right)\left[F\left(x_{n}\right)\right]^{k-1} f\left(x_{n}\right), \\
x_{1}>x_{2}>\ldots>x_{n} .
\end{array}
$$

The $p d f$ of $Y_{n}^{(k)}$ and the joint $p d f$ of $Y_{m}^{(k)}$ and $Y_{n}^{(k)}$ are given respectively by

$$
\begin{gathered}
f_{Y_{n}^{(k)}}(x)=\frac{k^{n}}{(n-1) !}[-\ln F(x)]^{n-1}[F(x)]^{k-1} f(x), n \geq 1 \\
f_{Y_{m}^{(k)}, Y_{n}^{(k)}}(x, y)=\frac{k^{n}}{(m-1) !(n-m-1) !}[-\ln F(x)]^{m-1} \\
\times[\ln F(x)-\ln F(y)]^{n-m-1} \frac{f(x)}{F(x)}[F(y)]^{k-1} f(y), x>y, \\
1 \leq m<n, \quad n \geq 2 .
\end{gathered}
$$

Further, for lower record values, the conditional $p d f$ of $Y_{n}^{(1)}$ given $Y_{m}^{(1)}=x$, $1 \leq m \leq n$ in view of (2) and (3) for $k=1$ is

$$
f_{Y_{n}^{(1)} \mid Y_{m}^{(1)}=x}(y)=\frac{1}{(n-m-1) !}[-\ln F(y)+\ln F(x)]^{n-m-1} \frac{f(y)}{F(x)}, x>y .
$$

A random variable $\mathrm{X}$ is said to have exponentiated Burr XII (EB XII) distribution if its $p d f$ is of the form

$$
f(x)=\alpha \beta \theta x^{\alpha-1}\left(1+x^{\alpha}\right)^{-(\theta+1)}\left[1-\left(1+x^{\alpha}\right)^{-\theta}\right]^{\beta-1}, \quad x>0, \quad \alpha, \beta, \theta>0
$$

and the corresponding $c d f$ is given, by

$$
F(x)=\left[1-\left(1+x^{\alpha}\right)^{-\theta}\right]^{\beta}, x>0, \alpha, \beta, \theta>0 .
$$

Further, the survival function and hazard rate function of $E B X I I$ distribution are given, respectively by

$$
S(x)=1-\left[1-\left(1+x^{\alpha}\right)^{-\theta}\right]^{\beta}, x>0, \alpha, \beta, \theta>0
$$

and

$$
h(x)=\frac{\alpha \beta \theta x^{\alpha-1}\left(1+x^{\alpha}\right)^{-\theta-1}\left[1-\left(1+x^{\alpha}\right)^{-\theta}\right]^{\beta-1}}{\left[1-\left\{1-\left(1+x^{\alpha}\right)^{-\theta}\right\}^{\beta}\right]} .
$$

The paper is organized as follows. Section 2 gives explicit expressions and recurrence relations for single moments of $k$-th lower record values from exponentiated Burr XII distribution. The obtained relations are used to compute first four moments, variance, skewness, kurtosis and coefficient of variation of lower record values. In Section 3, explicit expressions and recurrence relations for product moments of $k$-th lower record values from 
exponentiated Burr XII distribution are derived. Further, in Section 4, two characterization theorems of this distribution are also obtained on using a recurrence relation for single moments and conditional expectation of record values. Also, maximum likelihood estimates of the parameters of exponentiated Burr XII distribution based on $k$-th lower record valuesare derived and the confidence intervals using Fisher information matrix are obtained in Section 5. Section 6 consists of simulation study based on the maximum likelihood estimates of the parameters of exponentiated Burr XII distribution based on lower record values. In Section 7, a case study is provided to illustrate the performance of maximum likelihood estimates of exponentiated Burr XII distribution.

\section{Relations for single moments}

In the section, we obtain the explicit expressions and recurrence relations for the single moments of the $k$-th lower record values from the EB XII distribution.

Note that, the marginal distribution of lower record values is

$$
\begin{aligned}
f_{Y_{n}^{(x)}} & =\frac{\alpha \theta(\beta k)^{n}}{(n-1) !} x^{\alpha-1}\left(1+x^{\alpha}\right)^{-\theta-1}\left[-\ln \left\{1-\left(1+x^{\alpha}\right)^{-\theta}\right\}\right]^{n-1} \\
\quad \times & {\left[1-\left(1+x^{\alpha}\right)^{-\theta}\right]^{\beta k-1} . }
\end{aligned}
$$

Let us denote the r-th moment of $Y_{n}^{(k)}$ by $\mu_{L(n): k}^{(r)}$. Similarly, let $\mu_{L(m, n): k}^{(r, s)}$ denote the $(r, s)$-th product moment of $Y_{m}^{(k)}$ and $Y_{n}^{(k)}$.

$$
\mu_{L(n): k}^{(r)}=\frac{k^{n}}{(n-1) !} \int_{0}^{\infty} x^{r}[-\ln (F(x))]^{n-1}[F(x)]^{k-1} f(x) d x .
$$

By substituting $t=[F(x)]^{1 / \beta}$ in (9), we get

$$
\begin{aligned}
\mu_{L(n): k}^{(r)}= & \frac{(k \beta)^{n}}{(n-1) !} \sum_{p=0}^{\infty} \sum_{q=0}^{\infty} \frac{(-1)^{p+r / \alpha} \Gamma(r / \alpha+1)(p / \theta)_{(q)}}{\Gamma(p+1) \Gamma(q+1) \Gamma(r / \alpha-p+1)} \\
& \times \int_{0}^{1} t^{k \beta+q-1}[-\ln t]^{n-1} d t .
\end{aligned}
$$

Again by putting $u=-\ln t$, we obtain

$$
\mu_{L(n): k}^{(r)}=\sum_{p=0}^{\infty} \sum_{q=0}^{\infty} \frac{(-1)^{p+r / \alpha} \Gamma(r / \alpha+1)(p / \theta)_{(q)}}{\Gamma(p+1) \Gamma(q+1) \Gamma(r / \alpha-p+1)}\left(\frac{k \beta}{k \beta+q}\right)^{n} .
$$

Remark 1: For $k=1$ in (11), we deduce the explicit expression for single moments of lower record values from the EB XII distribution. 
Theorem 1: For a positive integer $k \geq 1$ and for $n \geq 1$ and $r=0,1,2, \ldots \ldots$,

$$
\left(1+\frac{r}{\alpha \beta k}\right) \mu_{L(n): k}^{(r)}=\mu_{L(n-1): k}^{(r)}-\frac{r}{\alpha \beta \theta k} \sum_{u=2}^{\theta+1}\left(\begin{array}{c}
\theta+1 \\
u
\end{array}\right) \mu_{L(n): k}^{(r+\alpha(u-1))} .
$$

Proof: Clearly, We have from (5) and (6), we see that

$$
F(x)=\frac{1}{\alpha \beta \theta}\left\{\theta x+\sum_{u=2}^{\theta+1}\left(\begin{array}{c}
\theta+1 \\
u
\end{array}\right) x^{\alpha(u-1)+1}\right\} f(x) \text {. }
$$

Therefore, for $r=0,1,2, \ldots$, we have

$$
\mu_{L(n): k}^{(r)}=\frac{k^{n}}{(n-1) !} \int_{0}^{\infty} x^{r}[-\ln (F(x))]^{n-1}[F(x)]^{k-1} f(x) d x .
$$

Integrating by parts taking $[F(x)]^{k-1} f(x)$ for integration and the rest of the integrand for differentiation, we get

$$
\mu_{L(n): k}^{(r)}=\mu_{L(n-1): k}^{(r)}-\frac{r k^{n-1}}{(n-1) !} \int_{0}^{\infty} x^{r-1}[-\ln (F(x))]^{n-1}[F(x)]^{k} d x .
$$

the constant of integration vanishes since the integral considered in (14) is a definite integral. On using (13), we obtain

$$
\begin{aligned}
& \mu_{L(n): k}^{(r)}=\mu_{L(n-1): k}^{(r)}-\frac{r k^{n-1}}{\alpha \beta(n-1) !} \int_{0}^{\infty} x^{r}[-\ln (F(x))]^{n-1}[F(x)]^{k-1} f(x) d x \\
& -\frac{r k^{n-1}}{\alpha \beta \theta(n-1) !} \sum_{u=2}^{\theta+1}\left(\begin{array}{c}
\theta+1 \\
u
\end{array}\right) \int_{0}^{\infty} x^{r+\alpha(u-1)}[F(x)]^{k-1}[-\ln (F(x))]^{n-1} f(x) d x
\end{aligned}
$$

and hence the result given in (12).

Remark 2: For $k=1$ in (12), we deduce the recurrence relation for single moments of lower record values from the $E B X I I$ distribution.

Table 1:First four moments, variance, skewness, kurtosis and coefficient of variation of lower record values from equation (11) for $k=1$. The parameter values are taken $\alpha=2, \beta=1$ and $\theta=2$.

\begin{tabular}{cccccccc}
\hline$n$ & $r=1$ & $r=2$ & $r=3$ & $r=4$ & Variance & Skewness & Kurtosis \\
\hline 1 & 0.7854 & 1.0000 & 2.3521 & 24.631 & 0.3831 & 16.554 & 134.882 \\
2 & 0.3888 & 0.2274 & 0.1810 & 0.1901 & 0.0762 & 2.5179 & 7.9598 \\
3 & 0.2334 & 0.0867 & 0.0440 & 0.0285 & 0.0323 & 2.2578 & 6.5833 \\
4 & 0.1481 & 0.0379 & 0.0137 & 0.0064 & 0.0160 & 2.7393 & 7.0446 \\
5 & 0.0963 & 0.0176 & 0.0047 & 0.0017 & 0.0083 & 3.5843 & 8.3549 \\
\hline
\end{tabular}




\section{Relations for product moments}

In this section, we obtain the explicit expressions and recurrence relations for product moments of the k-th lower record values from the EB XII distribution.

The $(\mathrm{r}, \mathrm{s})$ th distribution of $Y_{m}^{(k)}$ and $Y_{n}^{(k)}, m<n$ is given by

$$
\begin{array}{r}
f_{Y_{m}^{(k)}, Y_{n}^{(k)}}(x, y)=\frac{(\alpha \theta)^{2} k^{n} \beta^{n-2}}{(m-1) !(n-m-1) !} \frac{x^{\alpha-1} y^{\alpha-1}\left[-\ln \left\{1-\left(1+x^{\alpha}\right)^{-\theta}\right\}\right]^{m-1}}{\left(1+x^{\alpha}\right)^{-\theta-1}\left(1+y^{\alpha}\right)^{-\theta-1}} \\
\times \frac{\left[\ln \left\{1-\left(1+x^{\alpha}\right)^{-\theta}\right\}-\ln \left\{1-\left(1+y^{\alpha}\right)^{-\theta}\right\}\right]^{n-m-1}\left[1-\left(1+y^{\alpha}\right)^{-\theta}\right]^{\beta k-1}}{\left[1-\left(1+x^{\alpha}\right)^{-\theta}\right]} .
\end{array}
$$

The explicit expression for the product moments of $\mathrm{k}$-th lower record values can be obtained as

$$
\mu_{L(m, n): k}^{(r, s)}=\frac{k^{n}}{(m-1) !(n-m-1) !} \int_{0}^{\infty} x^{r}[-\ln (F(x))]^{m-1} \frac{f(x)}{F(x)} G(x) d x,
$$

where

$$
G(x)=\int_{0}^{x} y^{s}[-\ln (F(y))+\ln (F(x))]^{n-m-1}[F(y)]^{k-1} f(y) d y .
$$

By setting $w=\ln (F(x))-\ln (F(y))$ in (16), we obtain

$$
G(x)=\Gamma(n-m) \sum_{p=0}^{\infty} \sum_{q=0}^{\infty} \frac{(-1)^{p+s / \alpha} \Gamma(s / \alpha+1)(p / \theta)_{(q)}}{\Gamma(p+1) \Gamma(q+1) \Gamma(s / \alpha-p+1)} \frac{(F(x))^{k+q / \beta}}{(k+q / \beta)^{n-m}} .
$$

On substituting the above expression of $G(x)$ in (15), we obtain

$$
\begin{aligned}
& \mu_{L(m, n): k}^{(r, s)}=\frac{k^{n}}{(m-1) !} \sum_{p=0}^{\infty} \sum_{q=0}^{\infty} \frac{(-1)^{p+s / \alpha} \Gamma(s / \alpha+1)(p / \theta)_{(q)}}{\Gamma(p+1) \Gamma(q+1) \Gamma(s / \alpha-p+1)(k+q / \beta)^{n-m}} \\
& \times \int_{0}^{\infty} x^{r}[-\ln (F(x))]^{m-1}(F(x))^{k+q / \beta-1} f(x) d x .
\end{aligned}
$$

By setting $t=[F(x)]^{1 / \beta}$ in (17), we get

$$
\begin{aligned}
& \mu_{L(m, n): k}^{(r, s)}=(k \beta)^{n} \sum_{p=0}^{\infty} \sum_{q=0}^{\infty} \sum_{v=0}^{\infty} \sum_{w=0}^{\infty} \frac{(-1)^{p+v+(r+s) / \alpha}(p / \theta)_{(q)}(v / \theta)_{(w)}}{\Gamma(p+1) \Gamma(q+1) \Gamma(v+1) \Gamma(w+1)} \\
& \times \frac{\Gamma(s / \alpha+1) \Gamma(r / \alpha+1)}{\Gamma(s / \alpha-p+1) \Gamma(r / \alpha-v+1)(k \beta+q)^{n-m}(k \beta+q+w)^{m}} .
\end{aligned}
$$

Remark 3: For $k=1$ in (18), we deduce the explicit expression for product moments of lower record values from the $E B X I I$ distribution.

Theorem 2: For $1 \leq m \leq n-2$ and $r, s=1,2, \ldots$,

$$
\left(1+\frac{s}{\alpha \beta k}\right) \mu_{L(m, n): k}^{(r, s)}=\mu_{L(m, n-1): k}^{(r, s)}-\frac{s}{\alpha \beta \theta k} \sum_{u=2}^{\theta+1}\left(\begin{array}{c}
\theta+1 \\
u
\end{array}\right) \mu_{L(m, n): k}^{(r, s+\alpha(u-1))} .
$$


Proof: For $1 \leq m \leq n-2$ and $r, s=1,2, \ldots$,

$$
\mu_{L(m, n): k}^{(r, s)}=\frac{k^{n}}{(m-1) !(n-m-1) !} \int_{0}^{\infty} x^{r}[-\ln (F(x))]^{m-1} \frac{f(x)}{F(x)} I(x) d x,
$$

where

$$
I(x)=\int_{0}^{x} y^{s}[-\ln (F(y))+\ln (F(x))]^{n-m-1}[F(y)]^{k-1} f(y) d y .
$$

Integrating $I(x)$ by parts taking $[F(y)]^{k-1} f(y)$ for integration and the rest of the integrand for differentiation and substituting the resulting expression in (4.6), we get

$$
\begin{aligned}
\mu_{L(m, n): k}^{(r, s)}= & \mu_{L(m, n-1): k}^{(r, s)}-\frac{s k^{n-1}}{(m-1) !(n-m-1) !} \int_{0}^{\infty} \int_{0}^{x} x^{r} y^{s-1}[-\ln (F(x))]^{m-1} \\
& \times[\ln (F(x))-\ln (F(y))]^{n-m-1}[F(y)]^{k} \frac{f(x)}{F(x)} d y d x,
\end{aligned}
$$

the constant of integration vanishes since the integral in $G(x)$ is a definite integral. On using the relation (13), we obtain

$$
\begin{aligned}
& \mu_{L(m, n): k}^{(r, s)}=\mu_{L(m, n-1): k}^{(r, s)}-\frac{s k^{n-1}}{\alpha \beta(m-1) !(n-m-1) !}\left[\int_{0}^{\infty} \int_{0}^{x} x^{r} y^{s}\right. \\
& \times[-\ln (F(x))]^{m-1}[\ln (F(x))-\ln (F(y))]^{n-m-1}[F(y)]^{k-1} \frac{f(x)}{F(x)} f(y) d y d x \\
& +\frac{1}{\theta} \sum_{u=2}^{\theta+1}\left(\begin{array}{c}
\theta+1 \\
u
\end{array}\right) \int_{0}^{\infty} \int_{0}^{x} x^{r} y^{s+\alpha(u-1)}[-\ln (F(x))]^{m-1} \\
& \left.\quad \times[\ln (F(x))-\ln (F(y))]^{n-m-1}[F(y)]^{k-1} \frac{f(x)}{F(x)} f(y) d y d x\right]
\end{aligned}
$$

and hence the result given in (19).

Remark 4: For $k=1$ in (19), we deduce the recurrence relation for product moments of lower record values from the EB XII distribution.

One can also note that Theorem 1 can be deduced from Theorem 2 by setting $r=0$ and replacing $s$ by $r$.

\section{Characterization}

This section contains characterizations of $E B$ XII distribution by using the recurrence relation for the moments of $k$-th lower record values and conditional expectation of lower record values. 
Let $L(a, b)$ stands for the space of all integrable functions on $(a, b)$. A sequence $\left\{f_{n}\right\} \subset L(a, b)$ is called complete on $L(a, b)$ if for all functions $g \in L(a, b)$ the condition

$$
\int_{a}^{b} g(x) f_{n}(x) d x=0, \quad n \in \mathrm{N},
$$

implies $g(x)=0$ a.e. on $(a, b)$. We start with the following result of Lin (1986).

Proposition 1: Let $n_{0}$ be any fixed non-negative integer, $-\infty<a<b<\infty$ and $g(x) \geq 0$, an absolutely continuous function, with $g^{\prime}(x) \neq 0$ a.e. on $(a, b)$. Then the sequence of functions $\left\{(g(x))^{n} e^{-g(x)}, n \geq n_{0}\right\}$ is complete in $L(a, b)$ iff $g(x)$ is strictly monotone on $(a, b)$.

Using the above Proposition we get a stronger version of Theorem 1.

Theorem 3: Let $k \geq 1$ be a fixed positive integer, $r$ be a non-negative integer and $X$ be an absolutely continuous random variable with $c d f F(x)$ and $p d f$ $f(x)$ on the support $(0, \infty)$, then

$$
\left(1+\frac{r}{\alpha \beta k}\right) \mu_{L(n): k}^{(r)}=\mu_{L(n-1): k}^{(r)}-\frac{r}{\alpha \beta \theta k} \sum_{u=2}^{\theta+1}\left(\begin{array}{c}
\theta+1 \\
u
\end{array}\right) \mu_{L(n): k}^{(r+\alpha(u-1))}
$$

if and only if

$$
F(x)=\left[1-\left(1+x^{\alpha}\right)^{-\theta}\right]^{\beta}, x>0, \alpha, \beta, \theta>0 .
$$

Proof: The necessary part follows immediately from equation (12). On the other hand if the recurrence relation in equation (21) is satisfied, then on using equation (1), we have

$$
\begin{aligned}
\frac{k^{n}}{(n-1) !} \int_{0}^{\infty} x^{r}[-\ln (F(x))]^{n-1}[F(x)]^{k-1} f(x) d x \\
=\frac{k^{n-1}}{(n-2) !} \int_{0}^{\infty} x^{r}[-\ln (F(x))]^{n-2}[F(x)]^{k-1} f(x) d x \\
\quad-\frac{r k^{n-1}}{\alpha \beta(n-1) !} \int_{0}^{\infty} x^{r}[-\ln (F(x))]^{n-1}[F(x)]^{k-1} f(x) d x \\
-\frac{r k^{n-1}}{\alpha \beta \theta(n-1) !} \sum_{u=2}^{\theta+1}\left(\begin{array}{c}
\theta+1 \\
u
\end{array}\right) \int_{0}^{\infty} x^{r+\alpha(u-1)}[-\ln (F(x))]^{n-1} \\
\times[F(x)]^{k-1} f(x) d x .
\end{aligned}
$$

Integrating the first integral on the right hand side of equation (22) by parts and simplifying the resulting expression, we find that 


$$
\begin{aligned}
& \frac{r k^{n-1}}{(n-1) !} \int_{0}^{\infty} x^{r-1}[-\ln (F(x))]^{n-1}[F(x)]^{k-1} \\
& \quad \times\left\{F(x)-\frac{x}{\alpha \beta} f(x)-\frac{1}{\alpha \beta \theta} \sum_{u=2}^{\theta+1}\left(\begin{array}{c}
\theta+1 \\
u
\end{array}\right) x^{\alpha(u-1)+1} f(x)\right\} d x=0 .
\end{aligned}
$$

It now follows from Proposition 5.1 that

$$
\frac{F(x)}{f(x)}=\frac{1}{\alpha \beta \theta}\left\{\theta x+\sum_{u=2}^{\theta+1}\left(\begin{array}{c}
\theta+1 \\
u
\end{array}\right) x^{\alpha(u-1)+1}\right\},
$$

which proves that

$$
F(x)=\left[1-\left(1+x^{\alpha}\right)^{-\theta}\right]^{\beta}, \quad x>0, \alpha, \beta, \theta>0 .
$$

Theorem 4: Let $\mathrm{X}$ be an absolutely continuous random variable with $c d f F(x)$ and $p d f f(x)$ on the support $(0, \infty)$. Then

$$
\begin{gathered}
E\left(X_{L_{n}} \mid X_{L_{m}}=x\right]=\beta^{n-m} \sum_{p=0}^{\infty} \sum_{q=0}^{\infty} \frac{(-1)^{p+\alpha} \Gamma(1 / \alpha+1)(p / \theta)_{(q)}}{\Gamma(p+1) \Gamma(q+1) \Gamma(1 / \alpha-p+1)} \\
\times \frac{\left[1-\left(1+x^{\alpha}\right)^{-\theta}\right]^{q}}{(\beta+q)^{n-m}},
\end{gathered}
$$

if and only if

$$
F(x)=\left[1-\left(1+x^{\alpha}\right)^{-\theta}\right]^{\beta}, \quad x>0, \alpha, \beta, \theta>0 .
$$

Proof: From (4), we have

$$
E\left[X_{L_{n}} \mid X_{L_{m}}=x\right]=\frac{1}{(n-m-1) !} \int_{0}^{x} y\left[\ln \frac{F(x)}{F(y)}\right]^{n-m-1} \frac{f(y)}{F(x)} d y .
$$

By setting $t=\ln \frac{F(x)}{F(y)}$ in (25), we get

$$
\begin{aligned}
& E\left[X_{L_{n}} \mid X_{L_{m}}=x\right] \\
& \quad=\frac{(-1)^{1 / \alpha}}{(n-m-1) !} \int_{0}^{\infty}\left[1-\left(1-e^{-t / \beta}\left(1-\left(1+x^{\alpha}\right)^{-\theta}\right)\right)^{-1 / \theta}\right]^{1 / \alpha} t^{n-m-1} e^{-t} d t .
\end{aligned}
$$

Simplifying the above expression, we derive the relation given in (24).

To prove the sufficiency part, we have from (24) and (25)

$$
\frac{1}{(n-m-1) !} \int_{0}^{x} y\left[\ln \frac{F(x)}{F(y)}\right]^{n-m-1} f(y) d y=F(x) H_{n}(x),
$$

where

$$
H_{n}(x)=\beta^{n-m} \sum_{p=0}^{\infty} \sum_{q=0}^{\infty} \frac{(-1)^{p+\alpha} \Gamma(1 / \alpha+1)(p / \theta)_{(q)}}{\Gamma(p+1) \Gamma(q+1) \Gamma(1 / \alpha-p+1)} \frac{\left[1-\left(1+x^{\alpha}\right)^{-\theta}\right]^{q}}{(\beta+q)^{n-m}} .
$$


Differentiating (26) both sides with respect to $x$, we get

$$
\frac{1}{(n-m-2) !} \int_{0}^{x} y\left[\ln \frac{F(x)}{F(y)}\right]^{n-m-2} \frac{f(x)}{F(x)} f(y) d y=F(x) H_{n}^{\prime}(x)+f(x) H_{n}(x)
$$

or

$$
\frac{f(x)}{F(x)}=\frac{H_{n}^{\prime}(x)}{H_{n-1}(x)-H_{n}(x)}
$$

which proves that

$$
F(x)=\left[1-\left(1+x^{\alpha}\right)^{-\theta}\right]^{\beta}, \quad x>0, \alpha, \beta, \theta>0 .
$$

\section{Parameter estimation}

\subsection{Maximum likelihood estimation}

In this section, we obtain the maximum likelihood estimators of the parameters $\alpha, \beta$ and $\theta$ of $E B$ XII distribution when the available data are lower record values. Let $X_{1}, X_{2}, \ldots, X_{n}$ be a sequence of i.i.d. random variables $c d f F(x)$ and $p d f$ on positive support. Let $Y_{n}=\min \left\{X_{1}, X_{2}, \ldots, X_{n}\right\}$ for $n \geq 1$. The observation $X_{j}, j \geq 1$, is a lower record value of this sequence, if it is greater than all preceding observations that is $Y_{j}<Y_{j-1}$ for $j>1$.

Suppose we observe $n$ lower record values denoted by, say, $\underline{x}=\left\{X_{L(1)}, X_{L(2)}, \ldots, X_{L(n)}\right\}$ from a sequence of i.i.d. random variables following $E B X I I$ distribution with $p d f$ (5). The likelihood function based on the random sample of size $n$ is obtained from

$$
L(\alpha, \beta, \theta \mid \underline{x})=f\left(x_{L(n)} ; \alpha, \beta, \theta\right) \prod_{i=1}^{n-1} \frac{f\left(x_{L(i)} ; \alpha, \beta, \theta\right)}{F\left(x_{L(i)} ; \alpha, \beta, \theta\right)} .
$$

By using (5), equation (27) can be rewritten as

$$
L(\alpha, \beta, \theta \mid \underline{x})=\alpha^{n} \beta^{n} \theta^{n} e^{\beta \ln \left[1-\left(1+x_{L(n)}^{\alpha}\right)^{-\theta}\right]} \prod_{i=1}^{n} \frac{x_{L(i)}^{\alpha-1}\left(1+x_{L(i)}^{\alpha}\right)^{-\theta-1}}{\left[1-\left(1+x_{L(i)}^{\alpha}\right)^{-\theta}\right]} .
$$

The log-likelihood function $l(\alpha, \beta, \theta \mid \underline{x})=\ln L(\alpha, \beta, \theta \mid \underline{x})$ is

$$
\begin{gathered}
l(\alpha, \beta, \theta \mid x)=n(\ln \alpha+\ln \beta+\ln \theta)+(\alpha-1) \sum_{i=1}^{n} \ln x_{L(i)}-(\theta+1) \sum_{i=1}^{n} \ln \left(1+x_{L(i)}^{\alpha}\right) \\
-\sum_{i=1}^{n} \ln \left[1-\left(1+x_{L(i)}^{\alpha}\right)^{-\theta}\right]+\beta \ln \left[1-\left(1+x_{L(n)}^{\alpha}\right)^{-\theta}\right] .
\end{gathered}
$$


We assume that the parameters $\alpha, \beta$ and $\theta$ are unknown. To obtain the normal equations for the unknown parameters, we differentiate (28) partially with respect to $\alpha, \beta$ and $\theta$ and equate to zero. The resulting equations are

$$
\begin{aligned}
& 0=\frac{\partial l}{\partial \alpha}=\frac{n}{\alpha}+\sum_{i=1}^{n} \ln x_{L(i)}-(\theta+1) \sum_{i=1}^{n} \frac{x_{L(i)}^{\alpha} \ln x_{L(i)}}{\left(1+x_{L(i)}^{\alpha}\right)} \\
& -\sum_{i=1}^{n} \frac{\theta\left(1+x_{L(i)}^{\alpha}\right)^{-\theta-1} x_{L(i)}^{\alpha} \ln x_{L(i)}}{\left[1-\left(1+x_{L(i)}^{\alpha}\right)^{-\theta}\right]}+\frac{\beta \theta\left(1+x_{L(n)}^{\alpha}\right)^{-\theta-1} x_{L(n)}^{\alpha} \ln x_{L(n)}}{\left[1-\left(1+x_{L(n)}^{\alpha}\right)^{-\theta}\right]}, \\
& 0=\frac{\partial l}{\partial \beta}=\frac{n}{\beta}+\ln \left[1-\left(1+x_{L(n)}^{\alpha}\right)^{-\theta}\right]
\end{aligned}
$$

and

$$
\begin{aligned}
& 0=\frac{\partial l}{\partial \theta}=\frac{n}{\theta}-\sum_{i=1}^{n} \ln \left(1+x_{L(i)}^{\alpha}\right)-\sum_{i=1}^{n} \frac{\left(1+x_{L(i)}^{\alpha}\right)^{-\theta} \ln \left(1+x_{L(i)}^{\alpha}\right)}{\left[1-\left(1+x_{L(i)}^{\alpha}\right)^{-\theta}\right]} \\
& +\frac{\beta\left(1+x_{L(n)}^{\alpha}\right)^{-\theta} \ln \left(1+x_{L(n)}^{\alpha}\right)}{\left[1-\left(1+x_{L(n)}^{\alpha}\right)^{-\theta}\right]},
\end{aligned}
$$

The solutions of the above equations are the maximum likelihood estimators of the EB XII distribution parameters $\alpha, \beta$ and $\theta$, denoted $\hat{\alpha}_{M L E}, \hat{\beta}_{M L E}$ and $\hat{\theta}_{M L E}$, respectively. As the equations expressed in (30), (31) and (32) cannot be solved analytically, one must use a numerical procedure to solve them.

\subsection{Approximate confidence intervals}

In this section, we present the asymptotic confidence intervals for the parameters of the $E B X I I$ distribution. For interval estimation and hypothesis testing on the model parameters, we require the $3 \times 3$ Fisher information matrix

$$
I^{-1}(\Theta)=\left(\begin{array}{rrr}
-\frac{\partial^{2} l(\alpha, \beta, \theta)}{\partial^{2} \alpha} & -\frac{\partial^{2} l(\alpha, \beta, \theta)}{\partial \alpha \partial \beta} & -\frac{\partial^{2} l(\alpha, \beta, \theta)}{\partial \alpha \partial \theta} \\
-\frac{\partial^{2} l(\alpha, \beta, \theta)}{\partial^{2} \beta} & -\frac{\partial^{2} l(\alpha, \beta, \theta)}{\partial \beta \partial \theta} \\
& -\frac{\partial^{2} l(\alpha, \beta, \theta)}{\partial^{2} \theta}
\end{array}\right)_{(\alpha, \beta, \theta)=(\hat{\alpha}, \hat{\beta}, \hat{\theta})}^{-1}
$$




$$
=\left(\begin{array}{ccc}
\operatorname{Var}(\alpha) & \operatorname{Cov}(\alpha, \beta) & \operatorname{Cov}(\alpha, \theta) \\
& \operatorname{Var}(\beta) & \operatorname{Cov}(\beta, \theta) \\
& & \operatorname{Var}(\theta)
\end{array}\right),
$$

where the elements are defined in Appendix A. The expectations in the Fisher information matrix can be obtained numerically. Let $\hat{\Theta}=(\hat{\alpha}, \hat{\beta}, \hat{\theta})^{T}$ be the maximum likelihood estimate of $\Theta=(\alpha, \beta, \theta)^{T}$. Under the usual regularity conditions and that the parameters are in the interior of the parameter space, but not on the boundary, we have: $\sqrt{n}(\hat{\Theta}-\Theta) \stackrel{d}{\longrightarrow} N_{3}\left(\underline{0}, I^{-1}(\Theta)\right)$, where $I(\Theta)$ is the expected Fisher information matrix. The asymptotic behaviour is still valid if $I(\Theta)$ is replaced by the observed information matrix evaluated at $\hat{\Theta}$, that is $I(\hat{\Theta})$ . The multivariate normal distribution $N_{3}\left(0, I^{-1}(\hat{\Theta})\right)$, where the mean vector $\underline{0}=(0,0,0)^{T}$, can be used to construct confidence intervals and confidence regions for the individual parameters and for the survival and hazard functions. The above approach which is used to derive approximate $100(1-\xi) \%$ two-sided confidence intervals for the parameters $\alpha, \beta$ and $\theta$ are given by:

$$
\begin{aligned}
& \hat{\alpha} \pm z_{\xi / 2} \sqrt{\operatorname{Var}(\hat{\alpha})}, \\
& \hat{\beta} \pm z_{\xi / 2} \sqrt{\operatorname{Var}(\hat{\beta})}
\end{aligned}
$$

and

$$
\hat{\theta} \pm z_{\xi / 2} \sqrt{\operatorname{Var}(\hat{\theta})}
$$

respectively, where $z_{\xi / 2}$ is the upper $(\xi / 2)^{t h}$ percentile of a standard normal distribution.

\section{Simulation study}

In this section, we examine the performance of maximum likelihood estimates for the parameters of $E B X I I$ distribution based on lower record values by conducting various simulations for different sizes $(n=5,10,15)$. We carry out 1000 iterations each for the true values of the parameters I: $\alpha=1, \beta=1, \theta=2$ and II: $\alpha=1, \beta=2, \theta=2$. Table 2 lists the means of MLEs of the three parameters along with the respective mean squared errors (MSE). From the results, we see that as the sample size $n$ increases, the mean estimates of the parameters tend to be closer to the true parameter values. We also see that MSEs of the MLEs of 
$\alpha, \beta$ and $\theta$ decreases as the sample size $n$ increases which quantifies the consistency of the estimation procedures.

Table 2 Monte Carlo simulation results: mean estimates and MSEs of $\hat{\alpha}, \hat{\beta}$ and $\hat{\theta}$.

\begin{tabular}{cccccc}
\hline & & & & & \\
$\mathrm{n}$ & Parameter & Mean & MSE & Mean & MSE \\
\hline \multirow{5}{5}{} & $\boldsymbol{\alpha}$ & 0.2856507 & 0.9318336 & 0.3381612 & 1.127984 \\
& $\beta$ & 0.2842037 & 0.9893721 & 0.3039655 & 3.397408 \\
& $\theta$ & 0.7120387 & 3.4946390 & 0.4602336 & 3.444630 \\
$\mathbf{1 0}$ & $\boldsymbol{\alpha}$ & 0.5629003 & 0.6682980 & 0.5542888 & 1.110543 \\
& $\beta$ & 0.3989100 & 0.8870512 & 0.3523907 & 3.206682 \\
& $\theta$ & 1.1500920 & 2.6165210 & 0.5421800 & 3.382426 \\
$\mathbf{1 5}$ & $\boldsymbol{\alpha}$ & 0.6204872 & 0.5573083 & 0.7237106 & 1.023590 \\
& $\beta$ & 0.4415545 & 0.8837439 & 0.4471304 & 3.246006 \\
& $\theta$ & 1.1649730 & 2.4881020 & 0.5798811 & 3.343053 \\
\hline
\end{tabular}

\section{Real data analysis}

To illustrate the result of this paper, we analyze a real data set. Table 8.1 represents Floyd river flood rates for the years 1935-1973 in Iowa, USA. Akinsete, et al. (2008) and Alzaatreh et al. (2012) studied these data using the beta Pareto (BP) and gamma Pareto (GP) distributions respectively.

Table 3Annual flood discharge rates of the Floyd river data

\begin{tabular}{lcccccccccc} 
Years & & & \multicolumn{8}{c}{ Flood discharge (ft3/s) } \\
$1935-$ & 1460 & 4050 & 3570 & 2060 & 1300 & 1390 & 1720 & 6280 & 1360 & 7440 \\
1944 & & & & & & & & & & \\
$1945-$ & 5320 & 1400 & 3240 & 2710 & 4520 & 4840 & 8320 & 13900 & 71500 & 6250 \\
1954 & & & & & & & & & & \\
$1955-$ & 2260 & 318 & 1330 & 970 & 1920 & 15100 & 2870 & 20600 & 3810 & 726 \\
1964 & & & & & & & & & & \\
$1965-$ & 7500 & 170 & 2000 & 829 & 17300 & 4740 & 13400 & 2940 & 5660 \\
1973 & & & & & & & & &
\end{tabular}

Form this data set, we extract the $n=4$ lower record values 1460, 1300, 318 and 170.Using the methods described in Section 5 we compute the maximum likelihood estimates as well as 95\% confidence interval for $\alpha$, $\beta$ and $\theta$ as given in Table 4 . 
Table 4MLE's and their confidence intervals

\begin{tabular}{cc}
\hline \multicolumn{2}{c}{ Data set } \\
\hline$\hat{\alpha}$ & 0.1782 \\
$\hat{\beta}$ & 2.1031 \\
$\hat{\theta}$ & 0.7393 \\
$C I(\hat{\alpha})$ & $(-0.019228,0.375683)$ \\
& \\
$C I(\hat{\beta})$ & $(-0.193245,4.399487)$ \\
& \\
$C I(\hat{\theta})$ & $(-0.5794162 .058032)$ \\
\hline
\end{tabular}

\section{Acknowledgements}

The authors would like to thank the reviewers and the editors for their comments which helped improve the paper 


\section{Appendix A}

Theelements of the Fisher information matrix $I(\Theta)$ for the parameters $(\alpha, \beta, \theta)$ are:

$$
\begin{aligned}
& \operatorname{Var}(\alpha)=-\frac{n}{\alpha^{2}}-(\theta+1) \sum_{i=1}^{n} \frac{x_{L(i)}^{\alpha}\left(\ln x_{L(i)}\right)^{2}}{\left(1+x_{L(i)}^{\alpha}\right)^{2}} \\
&-\theta \sum_{i=1}^{n} \frac{x_{L(i)}^{\alpha}\left(\ln x_{L(i)}\right)^{2}\left(1+x_{L(i)}^{\alpha}\right)^{-\theta-2}\left[1-\theta x_{L(i)}^{\alpha}-\left(1+x_{L(i)}^{\alpha}\right)^{-\theta}\right]}{\left[1-\left(1+x_{L(i)}^{\alpha}\right)^{-\theta}\right]^{2}} \\
&- \beta \theta \frac{x_{L(n)}^{\alpha}\left(\ln x_{L(n)}\right)^{2}\left(1+x_{L(n)}^{\alpha}\right)^{-\theta-2}\left[1-\theta x_{L(n)}^{\alpha}-\left(1+x_{L(n)}^{\alpha}\right)^{-\theta}\right]}{\left[1-\left(1+x_{L(n)}^{\alpha}\right)^{-\theta}\right]^{2}} \\
& \operatorname{Cov}(\alpha, \beta)=\frac{\theta x_{L(n)}^{\alpha} \ln x_{L(n)}\left(1+x_{L(n)}^{\alpha}\right)^{-\theta-1}}{\left[1-\left(1+x_{L(n)}^{\alpha}\right)^{-\theta}\right]} \\
& \operatorname{Cov}(\alpha, \theta)=-\sum_{i=1}^{n} \frac{x_{L(i)}^{\alpha} \ln x_{L(i)}}{\left(1+x_{L(i)}^{\alpha}\right)} \\
&-\sum_{i=1}^{n} \frac{x_{L(i)}^{\alpha} \ln x_{L(i)}\left(1+x_{L(i)}^{\alpha}\right)^{-\theta-1}\left[1-\left(1+x_{L(i)}^{\alpha}\right)^{-\theta}-\theta \ln \left(1+x_{L(i)}^{\alpha}\right)\right]}{\left[1-\left(1+x_{L(i)}^{\alpha}\right)^{-\theta}\right]^{2}} \\
&-\frac{\beta x_{L(n)}^{\alpha} \ln x_{L(n)}\left(1+x_{L(n)}^{\alpha}\right)^{-\theta-1}\left[1-\left(1+x_{L(n)}^{\alpha}\right)^{-\theta}-\theta \ln \left(1+x_{L(n)}^{\alpha}\right)\right]}{\left[1-\left(1+x_{L(n)}^{\alpha}\right)^{-\theta}\right]^{2}}
\end{aligned}
$$

$\operatorname{Var}(\beta)=-\frac{n}{\beta^{2}}$

$$
\begin{aligned}
& \operatorname{Cov}(\beta, \theta)=\frac{\left(1+x_{L(n)}^{\alpha}\right)^{-\theta} \ln \left(1+x_{L(n)}^{\alpha}\right)}{\left[1-\left(1+x_{L(n)}^{\alpha}\right)^{-\theta}\right]} \\
& \operatorname{Var}(\theta)=-\frac{n}{\theta^{2}}+\sum_{i=1}^{n} \frac{\left(1+x_{L(i)}^{\alpha}\right)^{-\theta}\left[\ln \left(1+x_{L(i)}^{\alpha}\right)\right]^{2}}{\left[1-\left(1+x_{L(i)}^{\alpha}\right)^{-\theta}\right]^{2}}
\end{aligned}
$$

$$
-\frac{\beta\left(1+x_{L(n)}^{\alpha}\right)^{-\theta}\left[\ln \left(1+x_{L(n)}^{\alpha}\right)\right]^{2}}{\left[1-\left(1+x_{L(n)}^{\alpha}\right)^{-\theta}\right]^{2}} \text {. }
$$




\section{References}

1. Ahsanullah, M. (1995): Record Statistics. Nova Science Publishers, New York.

2. Akinsete, A., Famoye, F. and Lee, C. (2008). The beta Pareto distribution. Statistics, 42, 547-563.

3. Alzaatreh, A., Famoye, F. and Lee, C. (2012). Gamma Pareto distribution and its applications. Journal of Modern Statistical Methods, 11, 78-95.

4. Balakrishnan, N. and Ahsanullah, M. (1995): Relations for single and product moments of record values from exponential distribution. J. Appl. Statist. Sci.,2, 73-87.

5. Chandler, K.N. (1952): The distribution and frequency of record values. J.Roy. Statist. Soc., Ser. B, 14, 220-228.

6. Feller, W. (1966): An Introduction to Probability Theory and its Applications, Volume 2. John Wiley and Sons, New York.

7. Glick, N. (1978): Breaking records and breaking boards. Amer. Math. Monthly, 85, 2-26.

8. Kumar, D. (2012): Recurrence relations for marginal and joint moment generating functions of generalized logistic distribution based on lower $k$ record values and its characterization. ProbStats Fouram, 5, 47-53.

9. Kumar, D. and Kulshrestha, A.(2013): Recurrence relations for moments of $k$-th record values from generalized beta distribution and a characterization. Journal of Statistics application in Probability, 2, 115-121.

10. Kumar, D.and Saran, J. (2014): Ratio and inverse moments of record values from Marshall-Olkin log-logistic distribution, Pacific Journal of Applied Mathematics.6, 11-21.

11. Kumar, D. (2015): Explicit Expressions and Statistical Inference of Generalized Rayleigh Distribution Based on Lower Record Values, Mathematical Methods of Statistics, 24, 225-241.

12. Kumar, D. Jain, N. and Gupta, S. (2015): The type I generalized half logistic distribution based on upper record values, Journal of Probability and Statistics, 2015, 01-11.

13. Kumar, D. (2016): $k$-th lower record values from of Dagum distribution, Discussiones Mathematicae Probability and Statistics, 36, 25-41. 
The Exponentiated Burr XII Distribution

14. Lin, G.D. (1986): On a moment problem. Tohoku Math. Journal, 38, 595598.

15. Nevzorov, V.B. (1987): Records. Theory probab. Appl., 32, 201-228.

16. Resnick, S.I. (1973): Record values and maxima. Ann. Probab., 4, 650-662.

17. Shorrock, R.W. (1973): Record values and inter record times. J. Appl. Probab., 10, 543-555. 
Devendra Kumar, Jagdish Saran and Neetu Jain 